\title{
Binary and Dimorphic: Composite and Discordant Lymphoma
}

\author{
Anubha Bajaj* \\ Department of Pathology, Panjab University, India \\ *Corresponding author: Anubha Bajaj, Department of Pathology, India
}

submission: December 4, 2018; Published: December 10, 2018

\begin{abstract}
Composite lymphoma as an entity was initially described by Custer et al followed by a modified delineation by Hicks et al and Kim et al with a categorical emergence of architecturally diverse and binary cytological subcategories of lymphoma within the same anatomic site or tissue. Composite lymphoma as a disorder essentially describes a biphasic elucidation of an identical malignant clone of cells [1]. A discordant lymphoma may be exemplified by a condition in which two disparate lymphomas may arise at different anatomic locations within an identical time frame (Figure 1). Sequential lymphoma may be characterized by the appearance of two distinctive histological variants of malignant lymphoma detected successively within a similar group of lymph nodes.
\end{abstract}

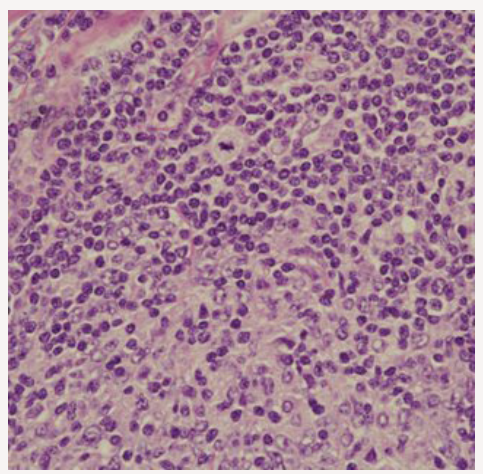

Figure 1: CL: small and large B cell zones with cellular pleomorphism [13].

\section{Preface}

The occurrence of two dissimilar categories of malignant lymphoma arising within an individual in a sequential manner or as a simultaneous event incriminating the aforementioned lymph node groups may be infrequently elucidated. The emergence of two distinct and well described categories oflymphoma appearing within a singular anatomic site or nodule may be designated as composite lymphoma [2,3]. The association of two divergent subtypes of lymphoma at concurrently emerging at several anatomic sites may be classified as discordant lymphoma (Figure 2). The concordant lymphomas may signify the appearance of two contradistinctive neoplasm either spontaneously or as a consequence of therapeutic intervention of a preceding malignancy. Majority of composite/ discordant lymphoma may signify the emergence of discrepant biologic and morphologic elucidation of the same lesion where the aggressive variant represents the histological manifestation of tumour progression. Composite lymphomas as an exceptional entity may account for an estimated $1 \%-4 \%$ of the de novo malignant lymphomas.

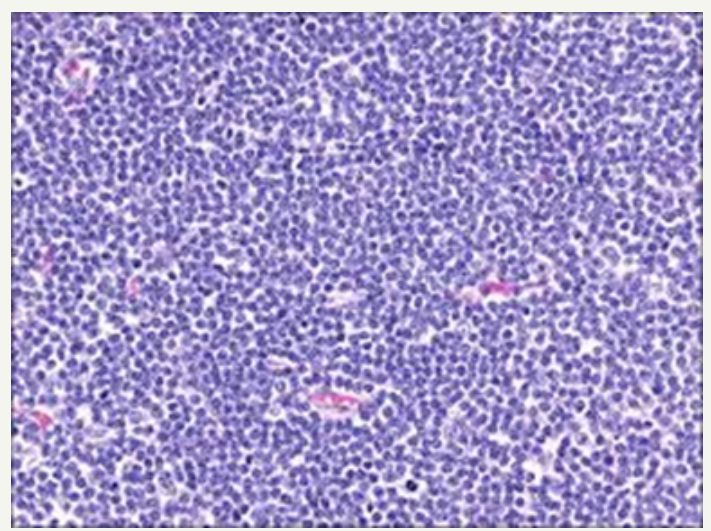

Figure 2: CL: peripheral T cell lymphoma and a small lymphocytic lymphoma [14].

\section{Disease Characteristics}

Two distinct lymphoid neoplasm situated in the same topographic location elucidating an identical time of presentation 
such as the composite lymphoma may progress in conjunction with concomitant categories of the tumour as described:

A. A low-grade B cell lymphoma (small lymphocytic, follicular or T cell rich B cell lymphoma) may transform into an aggressive diffuse large B cell lymphoma (DLBCL) [1].

B. A conversion of mantle cell lymphoma (MCL) into a higher-grade malignancy (blastic transformation).

C. A low-grade $\mathrm{T}$ cell lymphoma (such as mycosis fungoides) may modify into an aggressive, large $\mathrm{T}$ cell lymphoma.

D. A concurrence of nodular lymphocytic predominance Hodgkin's lymphoma (NLPHL) with adjunctive lymphomas especially the diffuse large B cell lymphoma (DLBCL) [1].

E. Concomitant appearance of classic Hodgkin's lymphoma and large B cell lymphoma. The dual malignancies may arise in the thymic zone. The Hodgkin's lymphoma may appear prior to, in concurrence with or follow the emergence of a non-Hodgkin's lymphoma.

F. A classic Hodgkin's lymphoma along with a follicular lymphoma may be a frequently configured composite lymphoma.

G. A classic Hodgkin's lymphoma may coexist with a peripheral (post thymic) T cell lymphoma. The particular tumours may be immune reactive to CD20+.

H. A classic Hodgkin's lymphoma may be commingled with foci of chronic lymphocytic leukaemia/ small lymphocytic lymphoma (CLL/SLL).

I. A classic Hodgkin's lymphoma may be admixed with a marginal zone B cell lymphoma.

J. A classic Hodgkin's lymphoma may transform into an anaplastic large cell lymphoma (ALCL).

K. Malignant lymphomas may be demarcated into categorical B lymphocyte and T lymphocyte zones [1]

L. A small lymphocytic lymphoma (SLL) may be expounded with a dendritic cell neoplasm.

\section{Disease Elucidation}

The majority of instances of composite lymphomas may elucidate binary forms of Non-Hodgkin's Lymphoma, predominantly comprising of mixed small and large cell lymphoma. The infrequent combination of a Hodgkin's lymphoma and NonHodgkin' Lymphoma confined to a singular lymph node may be considered as an amalgamation of disparate clinico-pathological entities [4-6] Composite lymphoma generally manifests as two divergent morphological lesions arising in a solitary lymph node. The histological variants may belong to an identical lineage of a $\mathrm{B}$ cell lymphoma, a subtype of a $\mathrm{T}$ cell lymphoma or an exceptional collocation of a B and a T lymphocyte tumour. Additionally, a composite lymphoma may signify a dual occurrence of Hodgkin's lymphoma and Non-Hodgkin's lymphoma in a singular lymph node (Figure 3).

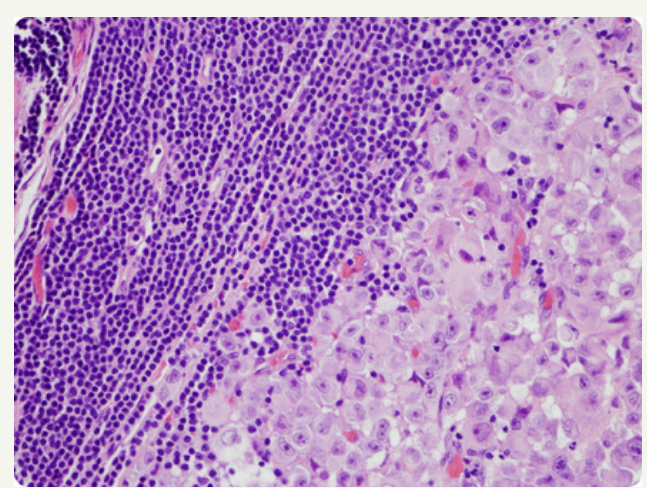

Figure 3: CL: a melanoma admixed with small lymphocytic lymphoma [15].

Definitive categories of a Non-Hodgkin's lymphoma may be witnessed with composite lymphoma. A mantle cell lymphoma and a follicular centre lymphoma defining the constituents of a composite lymphoma, as delineated by the immune histochemical and genetic studies, may elucidate divaricating cellular clones of origin. An immunoglobulin heavy chain (IgH) gene and BCL2 genetic rearrangement may be enunciated within the follicular centre cells. A duplicate clone of tumour cells may be exemplified on immune -histochemistry and genetic assay (Figure 4). Manual or laser capture micro-dissection in conjunction with multiple molecular methodologies for assessing the clone specific neoplasm, particularly the immunoglobulin heavy and light recombinant genetic sequence analysis may be employed [5,6].

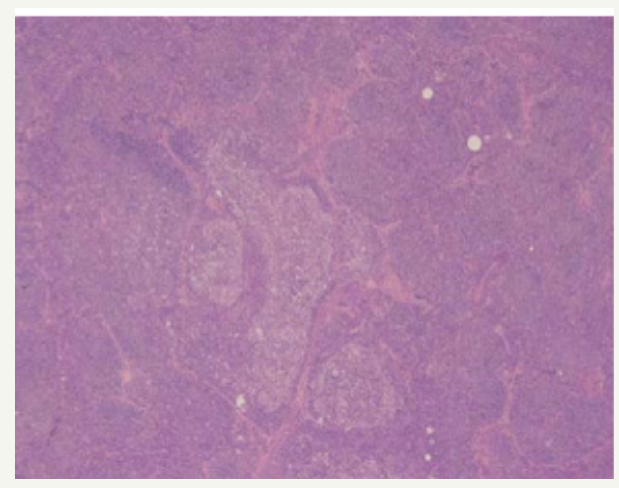

Figure 4: CL: nodular sclerosis Hodgkin's lymphoma with a follicular lymphoma [16].

Two dissimilar lymphomas elucidating a $t$ (14:18) and a $t$ (11:14) chromosomal translocation along with two diverse and clonally unrelated composites of malignant cells may be enunciated on immune histochemical, fluorescent in situ hybridization (FISH) and molecular methods. The assay of immunoglobulin light chain, especially the kappa chain may be beneficial for discerning clone specifications of the neoplasm.

A composite lymphoma comprising of a mantle cell lymphoma with a plasmacytoma may consistently be of a B cell lineage though a heterogeneous morphology may be depicted and an immune histochemical with a molecular confirmation may be mandated 
(Table 1). The histological evolution of a B cell lineage lymphoma may manifest in individuals with an initial diagnosis of a low-grade $B$ cell lymphoma such as a mantle cell lymphoma or a follicular centre cell lymphoma with the dissemination of miniature lymphocytes. Combinations of follicular small cleaved and follicular mixed small cleaved and large cell B cell lymphomas may be detected.

Table 1: Composite Lymphoma with Hodgkin's and Non-Hodgkin's Lymphoma variants [3].

\begin{tabular}{|c|c|}
\hline Non-Hodgkin's Lymphoma & Hodgkin's Lymphoma \\
\hline Large cell immunoblastic (75\%) & Nodular Sclerosis (25\%) \\
\hline Follicular and diffuse large cell (NA) & Unclassified (NA) \\
\hline Diffuse large cell (90\%) & Nodular Sclerosis (10\%) \\
\hline $\begin{array}{c}\text { Diffuse large cell (90\%) } \\
\text { Follicular and Diffuse large cell (50\%) }\end{array}$ & Mixed Cellularity (10\%) \\
\hline $\begin{array}{c}\text { Large cell Immunoblastic (75\%) } \\
\text { Diffuse Mixed Small and Large cell } \\
(95 \%)\end{array}$ & Mixed Cellularity (25\%) \\
\hline $\begin{array}{c}\text { Diffuse Large cell (50\%) } \\
\text { Unclassified (5\%) }\end{array}$ \\
\hline $\begin{array}{c}\text { Follicular Mixed Small cleaved and } \\
\text { Large cell (75\%) }\end{array}$ & Nodular Sclerosis (50\%) \\
\hline $\begin{array}{c}\text { Follicular Mixed Small cleaved and } \\
\text { Large cell (90\%) }\end{array}$ & Mixed Cellularity (10\%) \\
\hline Inter-follicular (10\%)
\end{tabular}

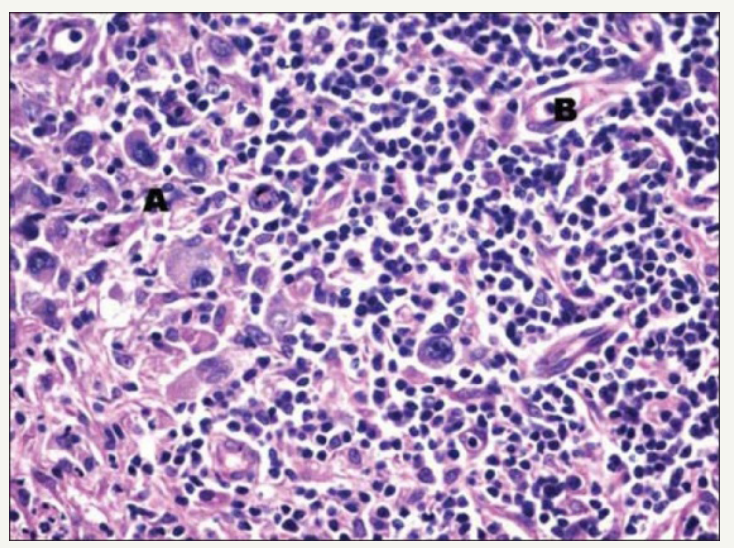

Figure 5: CL: a coexistent Hodgkin's lymphoma with a small lymphocytic lymphoma [17].

Composite lymphoma with coexistent Hodgkin's lymphoma with Non-Hodgkin's lymphoma may frequently emerge as a diffuse large B cell lymphoma (DLBCL) and a nodular lymphocyte predominance Hodgkin's lymphoma (NLPHL) (Figure 5).
Thus, the nodular tumour fraction (NLPHL) of the composite lymphoma indicates the low-grade component and a progression to a large B cell lymphoma, as may be evidenced by the immune histochemical elucidation of the Reed Sternberg (RS) cells confined to the lymphocyte predominant (NLPHL) subsection which may be immune reactive for $\mathrm{B}$ cell markers $[7,8]$. A heterogeneous manifestation of B cell markers localized within the components of diffuse large B cell lymphoma (DLBCL)and nodular lymphocyte predominance Hodgkin's lymphoma (NLPHL) may arise, although all $(100 \%)$ of the nodular (NLPHL) fraction may delineate a B cell phenotype.

The frequent concordance of Hodgkin's lymphoma and follicular lymphoma may necessitate further analysis. The Hodgkin's lymphoma may display a variable chromosomal translocation $t$ (14:18), discernible by a polymerase chain reaction (PCR). A high grade small non-cleaved cell non-Burkitt's lymphoma may appear within the peripheral lymph nodes, Waldeyer's ring and the bone marrow (Figure 6). Follicular low-grade lymphoma may further transform into a high grade small non-cleaved nonBurkitt's lymphoma. The low-grade lymphoma manifests genetic rearrangements of the BCL2 and c MYC gene with mutations of the p53 (tumour suppressor gene), which may usually be detected in the high grade small non-cleaved non Burkitt's lymphoma fraction. The specific molecular modifications may initiate the conversion of the lymphoma from a low-grade category to a high-grade category. A $\mathrm{T}$ cell lymphoma appearing subsequent to chemotherapy for Hodgkin's lymphoma may be elucidated, though a synchronous or a composite occurrence of the particular components may be infrequent. An exceptional combination of a Hodgkin's lymphoma with a $\mathrm{T}$ cell lymphoma may elucidate the systemic symptoms of a diffuse lymph node enlargement, fever, weight loss and night sweats (Figure 7). The lymph node subsequently examined may depict the emergence of a composite lymphoma constituted by a classic Hodgkin's lymphoma (c HL) and a peripheral T cell lymphoma (PTCL) [9]. Extra-nodal manifestations of the composite lymphoma may be delineated. A fluorescent in situ hybridization (FISH) technique may expound the presence of Epstein Barr Virus (EBV) in the Reed Sternberg cells.

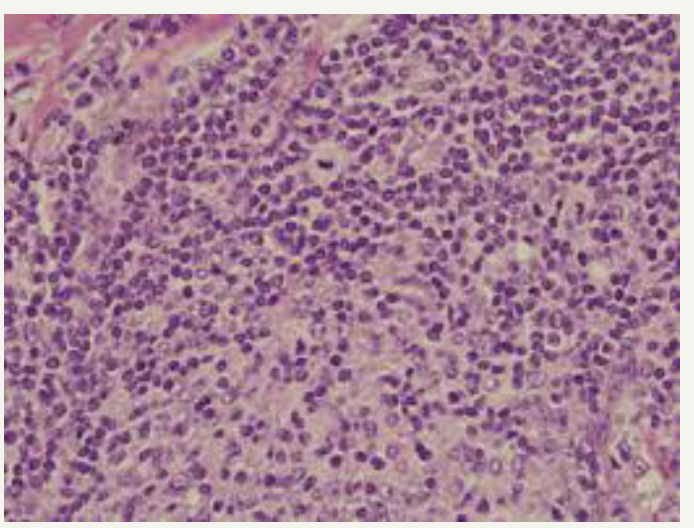

Figure 6: CL: small, malignant lymphocytes with enlarged, pleomorphic cells [16]. 


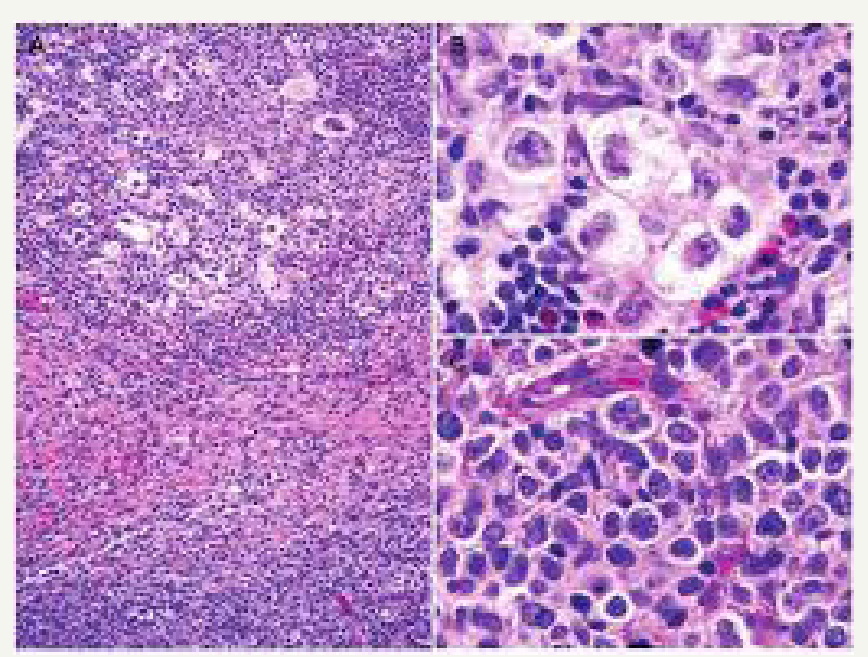

Figure 7: CL: nodular sclerosis Hodgkin's lymphoma and primary mediastinal large B cell lymphoma [13].

A low grade small cleaved lymphoma may undergo a mutation within the p53 gene which may transform it into a higher grade small non-cleaved non Burkitt's lymphoma. BCL2 genetic rearrangements detected within the low-grade component may authenticate the premise that small cleaved lymphoma may be generated from the follicular centre cells. Adjunctively, a follicular centre cell (FCC) lymphoma may be discerned within the lymphoid follicles of the tonsils or the lymph node. A small non-cleaved cell non Burkitt's lymphoma delineating an immune histochemical BCL2 genetic rearrangement may be consistent with the standardized configuration of BCL2 gene rearrangements (Figure 8). Small noncleaved non Burkitt's lymphoma may inconsistently demonstrate the BCL2 gene along with relevant rearrangements. An extranodal disease or a peripheral lymph node enlargement associated with BCL2 rearrangements may be exhibited with this particular lymphoma which originates from follicular centre cells (FCCs).

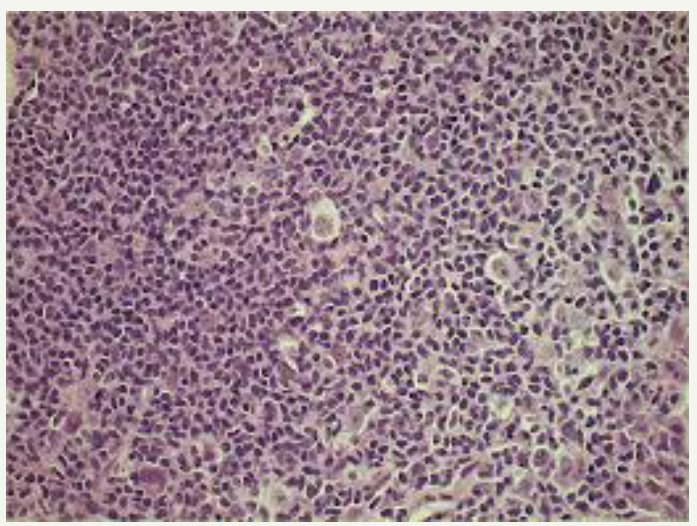

Figure 8: CL: a Hodgkin's lymphoma with diffuse large B cell lymphoma [18].

A transformation of a slow growing Non-Hodgkin's lymphoma to an aggressive histological variant, chiefly a diffuse large B cell lymphoma (DLBCL) may be eliminated from the category of composite lymphomas, though the dual components may appear at a similar location [10]. However, a composite lymphoma comprising of a small lymphocytic lymphoma/chronic lymphocytic leukaemia (SLL/CLL), a follicular lymphoma (FL) or a high-grade lymphoma with MYC and BCL2 genetic recombination (double hit lymphomas) may be detected at a single locus. The coexistence of a small lymphocytic lymphoma/ chronic lymphocytic leukaemia (SLL/CLL) with a follicular lymphoma (FL) may elucidate an immunoglobulin heavy chain $(\mathrm{IgH})$ rearrangement with concomitant BCL2 genetic fusion. This may be demonstrated by a fluorescent in situ hybridization (FISH) which exhibits the diffuse large cell (DLBCL) component directly evolving from the follicular lymphoma (FL).

The genetic amalgamation of immunoglobulin heavy chain (IgH) rearrangement with BCL2 recombination may be localized within the enlarged cells of composite lymphoma- a follicular lymphoma and a diffuse lymphoma (DLBCL) constituting the condition. (Figure 9) A fluorescent in situ hybridization (FISH) may demonstrate the MYC gene rearrangements detected within the diffuse lymphoma (DLBCL), which may be lacking in the follicular lymphoma (FL) component of the composite admixture. Nevertheless, a MYC gene rearrangement may be evidenced within an estimated 25\% $-50 \%$ of the follicular lymphoma (FL), whereas majority of the genetic recombination arises during the transformation of lymphoma to the aggressive variant. Notwithstanding, a MYC gene rearrangement may be detected in a mere $5 \%-10 \%$ of the de novo diffuse lymphomas (DLBCL). The acquired MYC gene rearrangements may objectively induce the transformation of follicular lymphoma to a diffuse lymphoma (DLBCL). A diffuse lymphoma (DLBCL) concurrently demonstrating immunoglobulin heavy chain (IgH), BCL2 and MYC gene rearrangements may be denominated as high-grade B cell lymphoma with MYC and BCL2 rearrangements (double hit lymphoma) and may generally be associated with an inferior prognosis.

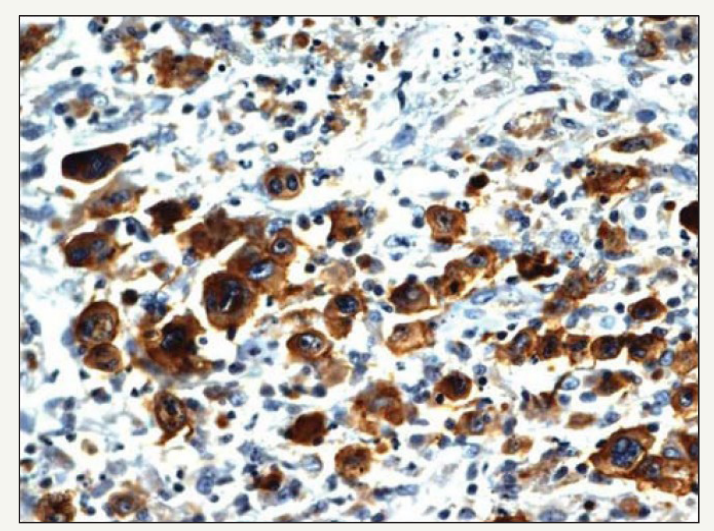

Figure 9: CL: a CD20+ immune reactivity with Hodgkin's lymphoma [17].

Composite lymphomas may be detected in conjunction with a follicular lymphoma, a small lymphocytic lymphoma/ chronic lymphocytic leukaemia (SLL/CLL) and a high-grade B cell lymphoma delineating MYC and BCL2 genetic rearrangements as detected on a polymerase chain reaction (PCR). The assay of the primary gene for immunoglobulin heavy chain (IgH) locus may exemplify disparate cellular clones and genetic rearrangements for the follicular lymphoma and small lymphocytic lymphoma/chronic 
lymphocytic leukaemia fractions, thereby indicating that the binary cellular components may emerge from two distinct clone's ab initio or may have digressed prior to the immunoglobulin heavy chain (IgH) gene rearrangement.

A fluorescent in situ hybridization (FISH) may be employed to demonstrate a divaricating cellular clone for the immunoglobulin heavy chain $(\mathrm{IgH})$ and BCL2 genes with a consequent genetic fusion. The attribute may appear within the follicular lymphoma though it may be lacking in the small lymphocytic lymphoma/ chronic lymphocytic leukaemia (SLL/CLL) component (Figure 10). The polymerase chain reaction (PCR) assay of immunoglobulin heavy chain (IgH) gene may signify an identical clone of cellular genesis betwixt the follicular lymphoma and small lymphocytic lymphoma component of the composite lymphoma. Thus, it may be conclusively stated that a disparate cellular clone may engender a follicular lymphoma and a small lymphocytic lymphoma/chronic lymphocytic leukaemia (SLL/CLL) within a composite lymphoma.

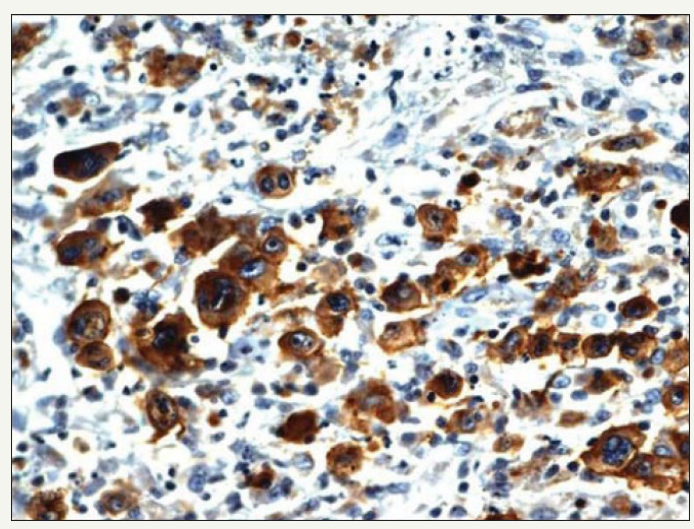

Figure 10: CL: immune reactive LMP1+ in Hodgkin's lymphoma with small lymphocytic lymphoma [17].

A primary splenic lymphoma may be an exceptional neoplasm collocating an estimated $<2 \%$ of the malignant lymphomas. The splenic disorder may be categorized as a diffuse lymphoma (DLBCL) or a low-grade lymphoma with a probable lymph node enlargement of the splenic hilum. The disorder may be designated as a primary splenic lymphoma if the tumour is restricted to the spleen with a lymph node enlargement confined to the hilum of the spleen and an absence of systemic lymph node enlargement or a liver incriminated by the lymphoma. A duration of six months or beyond may be mandated for the appearance of splenic lymphoma followed by a secondary lymphoma arising at an adjunctive location. Primary splenic lymphoma may delineate a splenomegaly, a bi or a pancytopenia with an absence of peripheral lymph node enlargement.

A composite lymphoma incriminating a primary splenic lymphoma may elucidate a BCL6 genetic rearrangement in an estimated one third (30\%) of cells. An immune reactive CD23+ may aid the detection of infrequent remnants of the residual lymphoid follicles. Germinal centres comprising of follicular dendritic cells (FDCs) may be enunciated and recognized by immune reactive PD-
1+ and CXCL13+. A CD5+ reactive component may be exemplified by the small interstitial lymphocytes. The enlarged B lymphocytes may manifest an immune reactive MUM1+ and Ki67+, thereby constituting an estimated half (50\%) of the CD20+ reactive congregation. A diffuse lymphoma (DLBCL) with a concomitant classic Hodgkin's lymphoma and an aggressive, undifferentiated component of a B cell lymphoma unclassifiable with features intermediate between diffuse lymphoma (DLBCL) and a classic Hodgkin's lymphoma (c HL) may be enunciated (Figure 11). The individuals may demonstrate B symptoms such as night sweats, generalized lymph node enlargement, fever and weight loss greater than $10 \%$ in the preceding six months. A composite lymphoma comprising of primary mediastinal large $B$ cell lymphoma (PMBCL) and classic Hodgkin's lymphoma may be considered exceptional. The primary mediastinal B cell lymphoma (PMBCL) generally appears in the third or fourth decades of life, though it may implicate adolescents and young adults. Classically, PMBCL may be restricted to the mediastinum. The lymphoma may disseminate while detection along with the incrimination of extra-nodal locales such as liver, kidney and adrenal glands. A morphology constituted by medium to enlarged cells with abundant cytoplasm and a spherical to lobulated nucleus may categorize the lymphoma.

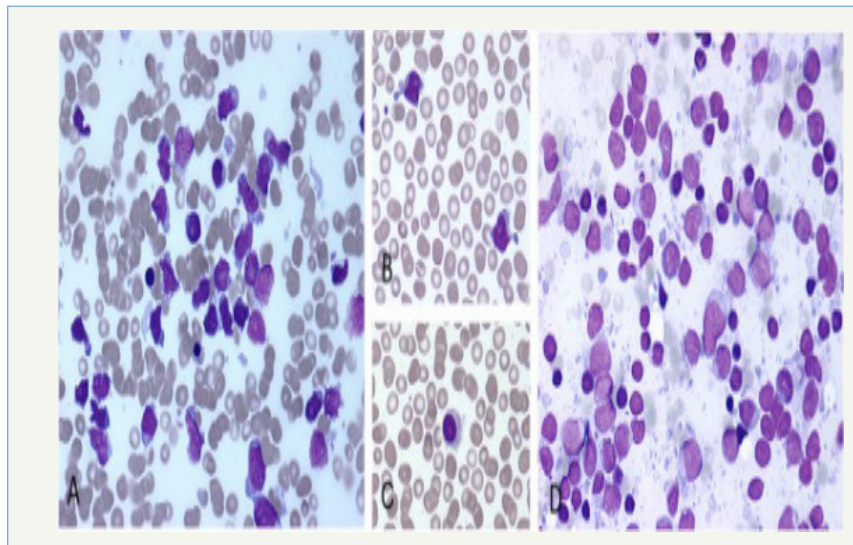

Figure 11: CL: a hepatopslenic T cell lymphoma with hairy cell leukemia [13].

Primary mediastinal large B cell lymphoma may elucidate B cell associated antigens particularly immune reactive CD19+, CD20+, CD22+ and CD79a+. The immune makers for a germinal centre origin (CD10+, BCL6+, MUM1-) may be expounded in a majority of PMBCL. The primary mediastinal large B cell lymphoma delineates several clinical, morphological and genetic attributes simulating the nodular sclerosis variant of Hodgkin's lymphoma. The lymphomas (PMBCL and NSHL) frequently detected in the young females may depict a common clinical exponent of a mediastinal mass. The infrequent, multinucleated malignant cells may be identical to the Reed Sternberg cells of Hodgkin's lymphoma. The gene expression profiling (GEP) analysis may depict a genotypic resemblance betwixt PMBCL and nodular sclerosis Hodgkin's lymphoma. An augmented REL proto oncogene along with a JAK2 tyrosine kinase gene may frequently be discerned with the neoplasm [11-18]. 


\section{Therapeutic Options}

Composite lymphoma is an infrequent disorder, so the therapeutic protocols may not be well delineated. With composite lymphoma comprising of Hodgkin's lymphoma and diffuse lymphoma (DLBCL), the therapy may be directed towards alleviating the high-grade histological component of the lymphoma. The therapeutic technique may be dictated by the subcategory of the composite lymphoma. Hodgkin's lymphoma may be managed concomitantly with an indolent B cell lymphoma where anti CD20 molecules such as rituximab may be employed either simultaneously or sequentially.

With a concomitant appearance of a Hodgkin's lymphoma with a diffuse lymphoma (DLBCL), an aggressive therapeutic combination may be recommended. Therapy may be initiated with the management of the higher grade of B cell lymphoma. The accepted norm for commencing therapy for diffuse lymphoma (DLBCL) is with the administration of rituximab, cyclophosphamide, doxorubicin, vincristine and prednisone (R CHOP). The double hit, aggressive lymphomas may elucidate an increased possibility of inadequate therapeutic outcomes. Intensified therapeutic regimen may be recommended in order to induce disease remission such as the employment of a dose adjusted etoposide, prednisone, vincristine cyclophosphamide, doxorubicin and rituximab (EPOCH $\mathrm{R})$. However, it may not be illustrated if the regimen is competent in augmenting the overall survival (OS) of the patients. The intensive chemotherapeutic regimen may be toxic which may restrict the applicability of the treatment with elderly individuals, particularly those elucidating co morbidities, as these may be frequent in individuals afflicted with composite lymphoma.

PMBCL may be appropriately managed by the initiation of dose adjusted etoposide, prednisone, vincristine cyclophosphamide, doxorubicin and rituximab (EPOCH R) which elucidates an overall survival of $97 \%$ with a median 5 year follow up. Hodgkin's lymphoma may be adequately treated with combinations of etoposide vincristine, cyclophosphamide, prednisone and doxorubicin and/or rituximab though bleomycin may be exempt in the lymphoma. A central nervous system (CNS) prophylaxis with the employment of intrathecal methotrexate may be a prerequisite on account of an elevated risk of tumour dissemination to the central nervous system with several adjunctive organs. EPOCH R may be an efficacious regimen in the management of a composite lymphoma comprising of primary mediastinal large $\mathrm{B}$ cell lymphoma (PMBCL) and Hodgkin's lymphoma. A consistent follow up may be necessitated in order to ascertain the durability of therapeutic decisions. The employment of radiotherapy may vary with the merits of the particular instance..

\section{References}

1. Rosai, Ackerman (1844) Surgical Pathology (10 $10^{\text {th }}$ edn).

2. Rami N, Siham F, Noha B, Hady G, Dany AG, et al. (2017) Composite hodgkin's and on hodgkin's lymphoma: Report of Two Cases with Unusual Presentations. Journal of Blood and Lymph 7(4): 180.

3. Fadwa J A (2007) Composite Lymphoma. JKAU Med Sci 14(1): 3-17.

4. Khan U, Hadid T, Ibrar W, Sano D, Al Katib A (2017) Composite lymphoma: opposite ends of spectrum meet. J Clin Med Res 9(3): 213-215.

5. Moore AM, Olga M, Gordon IS, Susan C, David PLB (2018) High grade B cell lymphoma with MYC and BCL2 re-arrangements arising in a composite lymphoma. Diagnostic Pathology 13(34): 1-4.

6. Kuppers R Dührsen U, Hansmann ML (2014) Pathogenesis diagnosis and treatment of composite lymphomas. Lancet Oncology 15(10): e435-e446.

7. Aukema SM, van Pel R, Nagel I, Bens S, Siebert R, et al. (2017) MYC expressions and translocation analyses in low grade and transformed follicular lymphoma. Histopathology 71(6): 960-971.

8. Abramson JS (2016) The spectrum of double hit lymphomas. Haematol Oncol Clin of North Am 30(6): 1239-1249.

9. Pasqualucci L, Khiabanian H, Fangazio M, Vasishtha M, Messina M, et al. (2014) Genetics of follicular lymphoma transformation. Cell Rep 6(1): 130-140.

10. Boiocchi L, Witter RE, He B, Subramaniyam S, Mathew S, et al. (2012) Composite chronic lymphocytic leukaemia/small lymphocytic lymphoma and follicular lymphoma are biclonal lymphomas: a report of two cases. Am J Clin Pathol 137(4): 647-659.

11. Koshy J, Dadfornia T, Qian YW (2014) Diffuse large B cell lymphoma arising in a composite lymphoma with biclonality by flow cytometry and one monoclonal band by PCR. Int J of Clin Exp Pathol 7(1): 407-410.

12. Dunleavy K, Stefania P, Lauren SM, Ranjana A, Clara CC, et al. (2013) Dose adjusted EPOCH- rituximab therapy in primary mediastinal B cell lymphoma. N Engl J Med 368(15): 1408-1416.

13. Courtesy: Research gate.

14. Courtesy: Sage Journals.

15. Courtesy: Blood advances.

16. Courtesy: Semantic scholar

17. Courtesy: Med IND.

18. Courtesy: Ingenta Connect.
Creative Commons Attribution 4.0 International License

For possible submissions Click Here

\section{Submit Article}

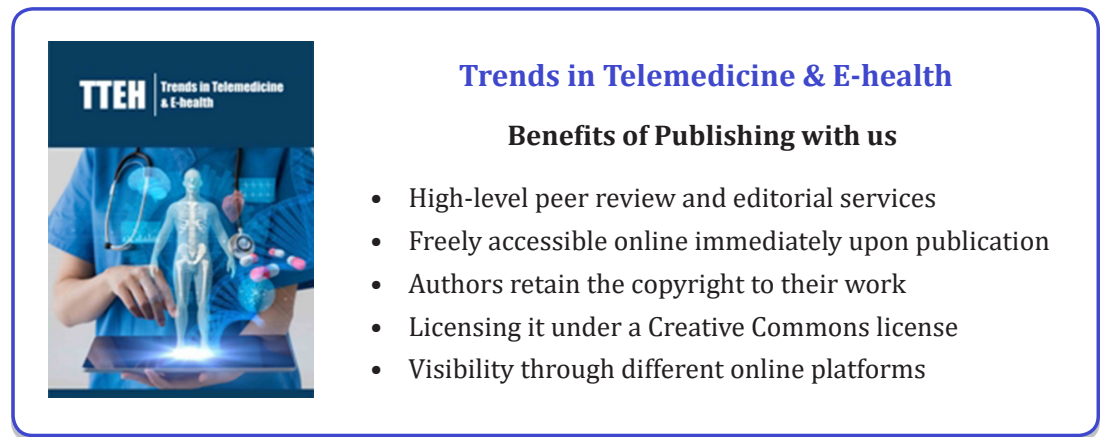

